\title{
Exogenous brain-derived neurotrophic factor attenuates neuronal apoptosis and neurological deficits after subarachnoid hemorrhage in rats
}

\author{
HUAYUN CHEN* ${ }^{*}$ YANWEI DANG ${ }^{*}$, XIAO LIU, JUNWEI REN and HONGQUAN WANG \\ Department of Neurosurgery, Xiangyang No. 1 People's Hospital Affiliated to Hubei \\ University of Medicine, Xiangyang, Hubei 441000, P.R. China
}

Received February 21, 2019; Accepted August 14, 2019

DOI: $10.3892 /$ etm.2019.8029

\begin{abstract}
Brain-derived neurotrophic factor (BDNF) is a growth factor crucial for neuronal survival, while its role in subarachnoid hemorrhage ( $\mathrm{SAH}$ )-induced neuronal apoptosis remains unclear. The aim of the present study was to investigate whether administering exogenous BDNF can protect against neuronal apoptosis and neurological deficits following $\mathrm{SAH}$ in a rat model. The BDNF level was found to be significantly decreased in the basal cortex at 6, 12, 24, 48 and $72 \mathrm{~h}$ following SAH. Exogenous BDNF significantly decreased the expression of Bax and reduced activation of caspase- 3 and caspase- 9 and the number of apoptotic neurons. Moreover, exogenous BDNF treatment significantly improved the neurological deficits at $72 \mathrm{~h}$ and long-term behavioral deficits (day 14) following SAH in a rat model. These findings indicate that exogenous BDNF attenuated $\mathrm{SAH}$-induced neuronal injury in rats.
\end{abstract}

\section{Introduction}

Subarachnoid hemorrhage (SAH) is associated with a 30-day mortality rate of $\sim 50 \%$ and is one of the most life-threatening cerebrovascular diseases (1). Deaths are mainly due to the initial hemorrhage and no effective treatment is currently available for brain injury (2). In recent years, neuronal death has been implicated in brain injury following experimental $\mathrm{SAH}$ and in a clinical setting, and is considered to play a crucial role in SAH-related brain injury $(1,2)$.

Correspondence to: Dr Hongquan Wang, Department of Neurosurgery, Xiangyang No. 1 People's Hospital Affiliated to Hubei University of Medicine, 15 Jiefang Road, Xiangyang, Hubei 441000, P.R. China

E-mail: hongquanwang2018@163.com

${ }^{*}$ Contributed equally

Key words: subarachnoid hemorrhage, brain-derived neurotrophic factor, neuronal apoptosis, neurological deficits
Brain-derived neurotrophic factor (BDNF) plays a key role in neuronal survival, synaptogenesis, neuronal differentiation and synaptic plasticity in the central nervous system (3-5). Clinical studies demonstrated that the BDNF Val66Met polymorphism is associated with poor recovery, but not associated with learning and memory performance following SAH in patients $(6,7)$. Animal studies demonstrated that the BDNF/tropomyosin-related kinase receptor B (TrkB) signaling pathway is involved in neuronal apoptosis following $\mathrm{SAH}(8,9)$. Expression of mature BDNF was found to be decreased at 4, 48 and $72 \mathrm{~h}$, and increased at 5 and 7 days post-SAH in a blood injection SAH model (10-12). Furthermore, recombinant human BDNF inhibited hemolysate-induced neuron death in an in vitro model of SAH (13). However, the role of BDNF in $\mathrm{SAH}-$ induced neuronal apoptosis and neurological deficits is unclear.

The aim of the present study was to investigate whether intracerebroventricular (ICV) injection of BDNF can protect against neuronal apoptosis and neurological deficits following $\mathrm{SAH}$ in a rat model.

\section{Materials and methods}

Experimental animals and design. A total of 117 male Sprague-Dawley rats (age, 12-weeks old; weight, 290-330 g) were obtained from the Animal Center of Xiangyang No. 1 People's Hospital (Xiangyang, China) and used according to the Guide of Care and Use of Laboratory Animals of the National Institutes of Health. All the animal experimental protocols were approved by the Animal Use and Care Committee of Hubei University of Medicine. The rats were group-housed with access to food and water ad libitum in a cage under controlled temperature $\left(25 \pm 2^{\circ} \mathrm{C}\right)$ and humidity $(55 \pm 10 \%)$ conditions, with a $12 \mathrm{~h}$-light/dark cycle. The experiment consisted of two parts: i) In order to investigate the time course of BDNF expression following SAH, 48 rats were subdivided into the sham and SAH groups at 6, 12, 24, 48 and $72 \mathrm{~h}$. Rats were decollated under deep anesthesia with pentobarbital sodium (40 mg/kg, i.p.) at each time point mentioned above. Then the basal cortex was collected for ELISA and western blot analysis $(n=6)$; ii) in order to investigate the role of exogenous BDNF in neuronal apoptosis after SAH, 69 rats 
were subdivided into sham, $\mathrm{SAH}+$ vehicle, and SAH + BDNF groups. The rats received a single ICV injection of BDNF at $30 \mathrm{~min}$ after SAH. Mortality was calculated at $72 \mathrm{~h}$. Following neurological assessment at $72 \mathrm{~h}$, rats were sacrificed under deep anesthesia with pentobarbital sodium (40 mg/kg, i.p.), 6 rats from each group were used for western blot analysis and 6 rats from each group were used for immunofluorescence staining. A total of 6 rats in each group were used for adhesive removal task and Frey test at day 14.

SAH model and grading system. The rat SAH model was constructed by the endovascular perforation method, as described previously $(14,15)$. Briefly, a 4-cm skin incision in the ventral neck was performed following anesthesia with pentobarbital sodium (40 mg/kg, i.p.) and the right common, external and internal carotid arteries were exposed. Subsequently, the external carotid artery was ligated and transected, and a blunted 4-0 monofilament nylon suture was inserted into the internal carotid artery for $18 \mathrm{~mm}$ and advanced a further $3 \mathrm{~mm}$ to perforate the wall of the bifurcation with the middle cerebral artery. The suture was removed after $10 \mathrm{sec}$, allowing reperfusion of the internal carotid artery. The sham-operated rats underwent an identical procedure, apart from the perforation. After completing the procedure, rats were arranged in a recovery cage for 30-60 min. After the rats started to eat some semi-fluid food and move around, they were housed in clean and new cages.

SAH grade score was obtained at $72 \mathrm{~h}$ using an 18-point SAH grading system (14). Briefly, rats were decollated under anesthesia with pentobarbital sodium $(40 \mathrm{mg} / \mathrm{kg}$, i.p.) and the brains were collected quickly. The SAH grade score was calculated according to the blood amount of six segments in the basal cistern. Each segment was scored (0-3) as follows: 0 , no subarachnoid blood; 1 , minimal subarachnoid blood; 2, moderate blood clot with visible arteries; and 3, blood clot covering all arteries. Total score was calculated by adding the scores from the six segments.

Drug administration. ICV injection was performed on experimental SAH rats as previously reported $(16,17)$. Briefly, the rats were fixed to the stereotactic frame after anesthesia with pentobarbital sodium (40 mg/kg, i.p.). Subsequently, a 30 -gauge needle of a Hamilton syringe was inserted into the left lateral ventricle via a burr hole $(-1.5 \mathrm{~mm}$ posterior, $-1.0 \mathrm{~mm}$ lateral and $-3.5 \mathrm{~mm}$ from bregma). The rats received ICV injection of recombinant rat BDNF protein $(10 \mu 1,0.5 \mathrm{nmol}$; cat. no. Ab9794; Abcam) or vehicle (10 $\mu \mathrm{l}$ PBS) at $30 \mathrm{~min}$ after SAH. After injection, the needle was kept for $5 \mathrm{~min}$ and then retracted slowly. The burr hole was quickly sealed with bone wax. Dosage drug administration was based upon prior research using an intracerebral hemorrhage rat model (18).

Western blot analysis. Western blotting was performed as described previously $(16,17)$. After sample preparation, $50 \mu \mathrm{g}$ protein of each sample was loaded onto an 12\% SDS-PAGE gel. After electrophoresis and transfer of proteins to a nitrocellulose membrane, the membranes were blocked at room temperature and incubated for $15 \mathrm{~h}$ at $4^{\circ} \mathrm{C}$ with the following primary antibodies: Anti-BDNF (1:1,000; cat. no. ab108319), anti-Bcl-2 (1:1,000; cat. no. ab59348), anti-Bax (1:1,000; cat. no. ab32503) and anti-activated caspase-3 (1:1,000; cat. no. ab2302), all from Abcam; anti-activated caspase-9 (1:1,000; cat. no. 9507) and anti- $\beta$-actin (1:1,000; cat. no. 4970), both from Cell Signaling Technology, Inc. Anti-rabbit or mouse IgG HRP-linked antibodies (all, 1:4,000; cat. no. 7074 and cat. no. 7076, respectively; Cell Signaling Technology, Inc.) were selected to incubate with the membrane at room temperature for $2 \mathrm{~h}$. The blots were visualized with ECL reagent (cat. no. 32106; Thermo Fisher Scientific, Inc.). The protein bands were selected to perform densitometry quantification with ImageJ software (v1.8; National Institutes of Health).

ELISA. To detect the level of BDNF, ELISA was performed as described previously $(12,19)$. The concentration of BDNF was measured with a rat BDNF ELISA kit (cat. no. ERBDNF; Thermo Fisher Scientific, Inc.) according to the manufacturer's protocol and was quantified as $\mu \mathrm{g} / \mathrm{g}$ tissue.

Modified Garcia score, rotarod test and beam balance test. The neurological deficits were assessed by an observer blinded to the experiment at $72 \mathrm{~h}$ after SAH using the modified Garcia score, beam balance test and rotarod test, as previously described $(14,15,20,21)$. The modified Garcia scoring system consisted of six tests that were scored as $0,1,2$ or 3 (no activity, spontaneous activity, spontaneous movements of all limbs and forelimb outstretching, respectively), and as 1, 2 or 3 (climbing wall of wire cage, body proprioception, and response to vibrissae touch, respectively). For the rotarod test, the rats were trained to stay on an accelerating rotarod (4-40 rpm until $5 \mathrm{~min}$, increasing by $4 \mathrm{rpm}$ per $30 \mathrm{sec}$ interval); three trials each day were performed for 1 week prior to SAH. The observer recorded the mean fall latency of each rat. For the beam balance test, the observer placed the rat on a $60-\mathrm{cm}$ square narrow wooden beam $(1 \mathrm{~cm}$ wide and $50 \mathrm{~cm}$ above the floor), and recorded the duration of the rat remaining on the center of the beam, until $60 \mathrm{sec}$ elapsed.

Immunohistochemical and Tunel staining. Activated caspase-3/NeuN immunostaining and Tunel staining were performed as described previously $(15,22)$. Briefly, frozen brain sections were fixed in $4 \%$ paraformaldehyde for $20 \mathrm{~min}$ at $4^{\circ} \mathrm{C}$, washed in PBS and then blocked in 5\% normal goat serum for $30 \mathrm{~min}$ at room temperature, and incubated for $15 \mathrm{~h}$ at $4^{\circ} \mathrm{C}$ with the primary antibodies: Anti-activated caspase- 3 (1:200; cat. no. ab2302; Abcam) and anti-NeuN (1:200; cat. no. ab177487; Abcam). The sections were washed in PBS and incubated for $2 \mathrm{~h}$ at room temperature with secondary antibodies: Fluorescein isothiocyanate-conjugated anti-rabbit IgG (1:400; cat. no. F9887; Sigma-Aldrich; Merck KGaA) and tetramethylrhodamine -conjugated anti-mouse IgG (1:400; cat. no. T5393; Sigma-Aldrich; Merck KGaA), then washed with PBS and a coverslip was added. For Tunel staining, the In situ Cell Death Detection kit with Fluorescein was used according to the manufacturer's protocol (Roche Diagnostics). Images were captured using a fluorescence microscope.

Adhesive removal task and Frey test. The long-term sensorimotor behavior deficit was assessed by an observer blinded to the experiment at day 14 after SAH using the adhesive removal task and Frey test, as described previously (23). Briefly, for the 

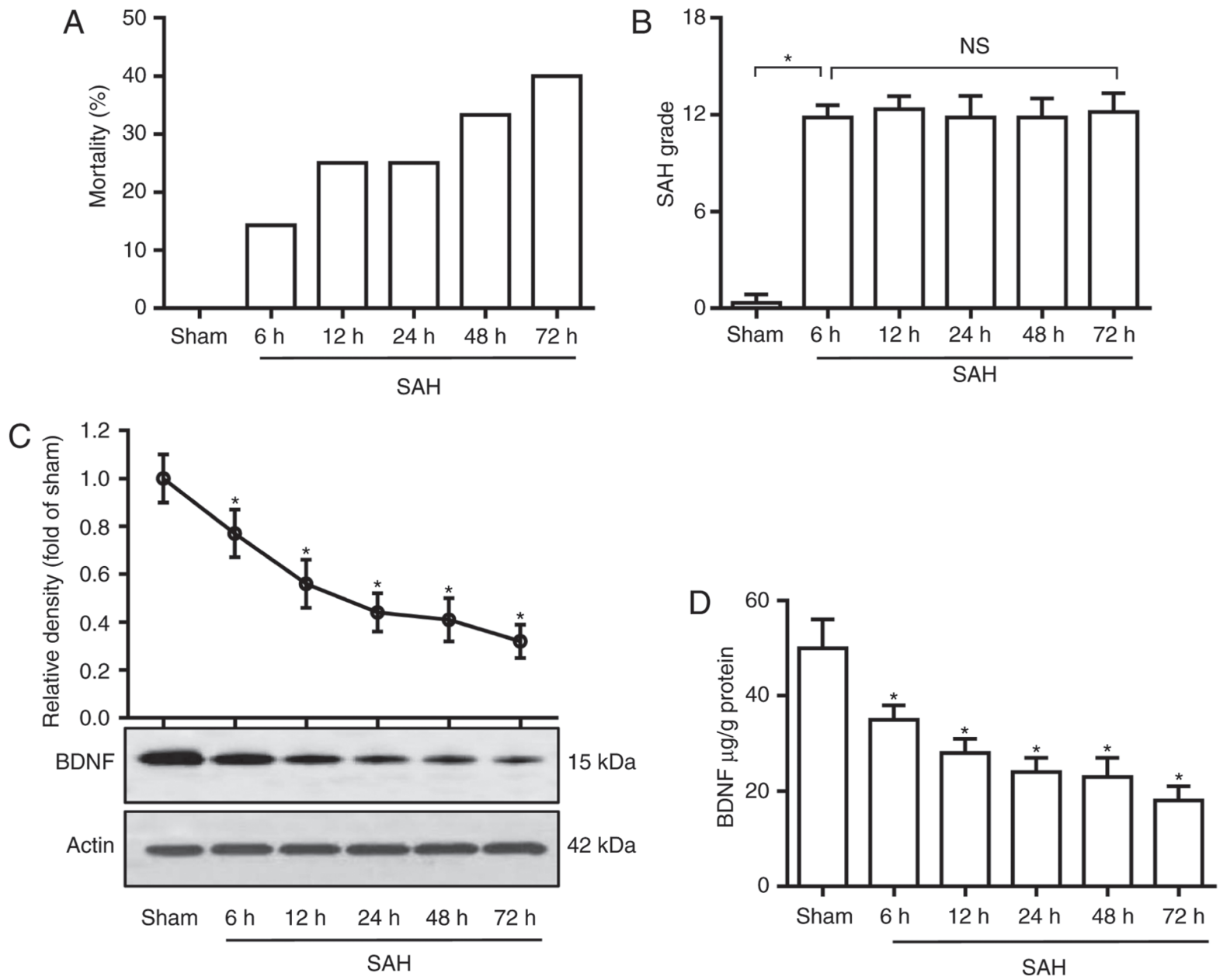

Figure 1. BDNF level decreases after SAH. (A) Mortality rate, (B) SAH grade scores, (C) western blot analysis of BDNF protein in basal cortex and (D) ELISA analysis of BDNF concentration in the basal cortex, in the sham and SAH groups at $6,12,24,48$ and 72 h. (B) $P=0.88$; (C and $D) P=0.01$. " $P<0.05$ vs. sham; one-way analysis of variance comparison test, $\mathrm{n}=6$ in each group. BDNF, brain-derived neurotrophic factor; $\mathrm{SAH}$, subarachnoid hemorrhage; ns, not significant.

adhesive removal task, the observer placed stickers on the right and left forepaws, then recorded the mean time of the three stickers' complete removal. The sticker placement on the forepaw was alternated. For the Frey test, the observer placed the rat on a wire grid bottom using the von Frey hairs (bending force 1-15 g). The Frey hair force was increased or decreased based on the rat response. The observer recorded mean time of the clear paw withdrawal, licking or shaking with increase in mechanical sensitivity and calculated the $50 \%$ paw withdrawal threshold.

Statistical analysis. All the data were presented as the mean \pm standard deviation obtained from 6 experimental repeats. The significance of the differences among multiple groups was analyzed by one-way analysis of variance followed by Dunn's post-hoc test in SPSS 16.0 statistical software (SPSS, Inc.). $\mathrm{P}<0.05$ was considered to indicate a statistically significant difference.

\section{Results}

SAH significantly decreases BDNF levels. The mortality rates were $0 \%$ ( 0 of 6 rats), $14.3 \%$ ( 1 of 7 rats), $25.0 \%$ ( 2 of 8 rats), $25.0 \%$ ( 2 of 8 rats), $33.3 \%$ (3 of 9 rats) and $40.0 \%$ (4 of 10 rats) in the sham and SAH groups at 6, 12, 24, 48 and $72 \mathrm{~h}$, respectively (Fig. 1A). The SAH grading scores were 11.8 \pm 0.7 , $12.3 \pm 0.8,11.9 \pm 1.3,11.8 \pm 1.1$ and $12.1 \pm 1.1$ in $\mathrm{SAH}$ groups at $6,12,24,48$ and $72 \mathrm{~h}$, respectively, there was no significant difference among the five groups (Fig. 1B). To determine the time course of BDNF expression following $\mathrm{SAH}$, rat basal cortices were dissected at 6, 12, 24, 48 and $72 \mathrm{~h}$ after SAH, and were assayed by western blot analysis and ELISA. As shown in Fig. $1 \mathrm{C}$ and D, SAH significantly decreased the BDNF level in the basal cortex compared with the sham group $(\mathrm{P}<0.05)$.

Exogenous BDNF attenuates neurological deficit at $72 \mathrm{~h}$ after $S A H$. The SAH grading scores were $12.3 \pm 1.0$ and $12.2 \pm 0.8$ in the $\mathrm{SAH}+$ vehicle and $\mathrm{SAH}+\mathrm{BDNF}$ groups, respectively. There was no significant difference between the two groups (Fig. 2A), indicating that the variation of SAH size did not affect the result. The mortality rates were $0 \%$ (0 of 18 rats), $33.3 \%$ (9 of 27 rats) and $25.0 \%$ (6 of 24 rats) in the sham, SAH + vehicle and SAH + BDNF groups, respectively (Fig. 2B). The modified Garcia score and the time on the rotarod and beam balance significantly decreased at $72 \mathrm{~h}$ in the $\mathrm{SAH}+$ vehicle group when compared with the sham group $(\mathrm{P}<0.05)$, suggesting that $\mathrm{SAH}$ caused neurological deficits and impaired the animals' ability to remain on the rotarod and 
A

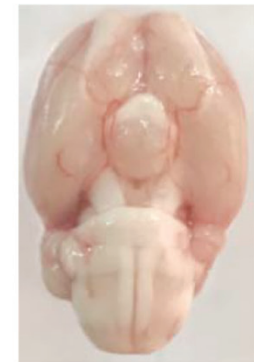

Sham

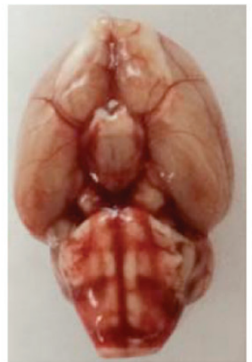

$\mathrm{SAH}+$ vehicle

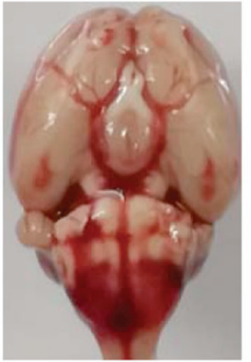

SAH+BDNF
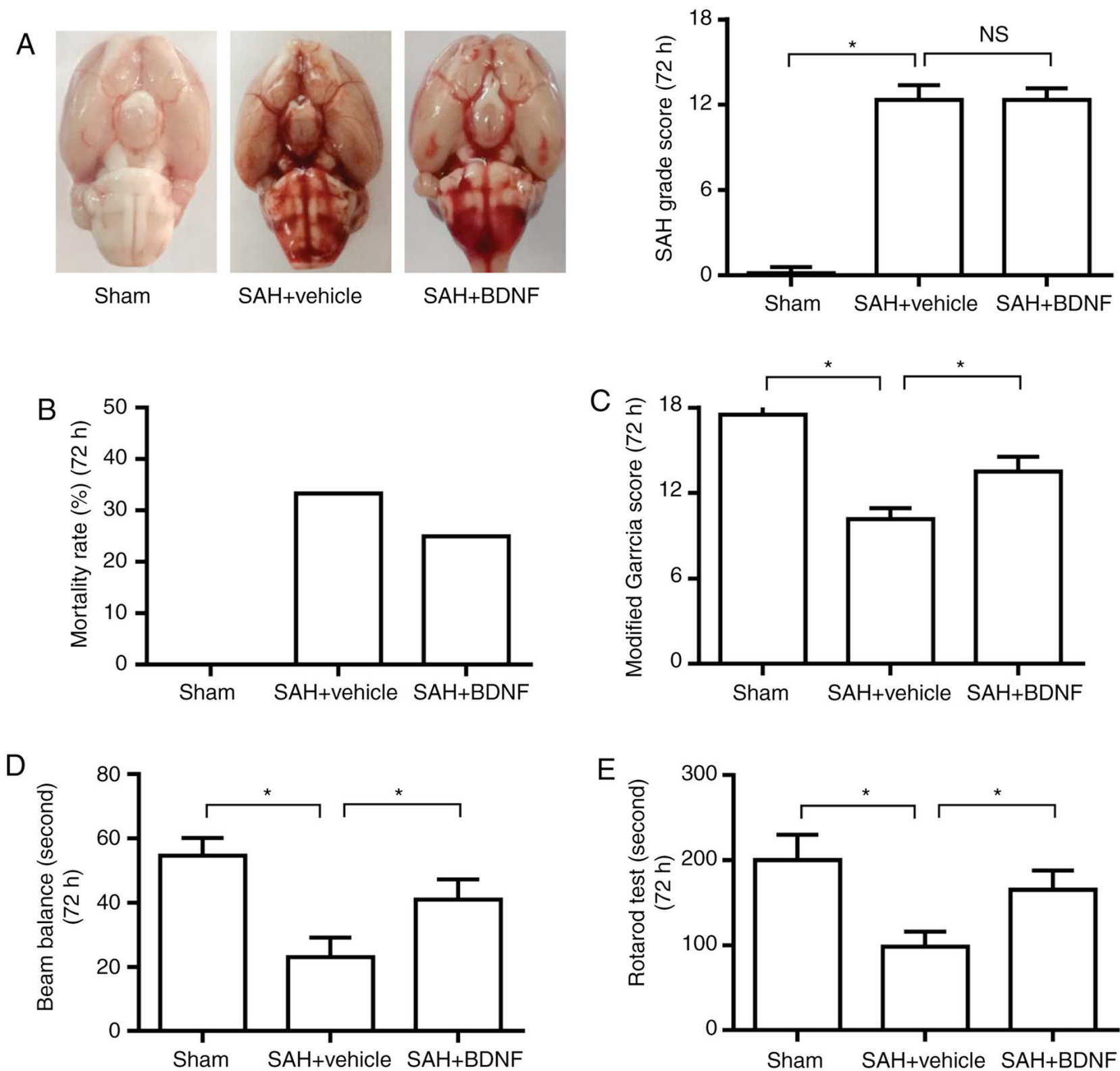

Figure 2. Exogenous BDNF attenuates neurological deficit at $72 \mathrm{~h}$ after SAH. (A) Representative brain photos of sham, SAH + vehicle and SAH + BDNF groups (left), SAH grade scores (right), (B) mortality rate, (C) modified Garcia score, (D) beam balance test score and (E) rotarod test score in the sham, SAH + vehicle and $\mathrm{SAH}+\mathrm{BDNF}$ groups. $\left(\mathrm{n}=6,{ }^{*} \mathrm{P}<0.05\right)$. (A) $\mathrm{P}=0.73$ and $(\mathrm{C}-\mathrm{E}) \mathrm{P}=0.01$. ${ }^{*} \mathrm{P}<0.05$; one-way analysis of variance comparison test, $\mathrm{n}=6$ in each group. BDNF, brain-derived neurotrophic factor; $\mathrm{SAH}$, subarachnoid hemorrhage.

beam (Fig. 2C-E). However, these neurobehavioral deficits improved with BDNF treatment compared with the SAH + vehicle group (Fig. 2C-E).

Exogenous BDNF attenuates neuronal apoptosis at $72 \mathrm{~h}$ after SAH. Cell apoptosis was assessed at $72 \mathrm{~h}$ using western blotting and immunofluorescence staining. As shown in Fig. 3, SAH caused marked downregulation of Bcl-2 and upregulation of Bax, activated caspase- 9 and activated caspase-3 (Fig. 3A and B). However, the expression of Bcl-2 markedly increased, whereas that of Bax, activated caspase- 9 and activated caspase- 3 notably decreased in the $\mathrm{SAH}+\mathrm{BDNF}$ group compared with the $\mathrm{SAH}+$ vehicle group (Fig. 3A and B). Furthermore, statistical analysis results revealed that SAH induction significantly increased the number of activated caspase-3- and TUNEL-positive neurons in the SAH + vehicle group compared with the sham group $(\mathrm{P}<0.001$; Fig. 3C and D), while BDNF treatment significantly reduced this increase $(\mathrm{P}<0.001)$.

Exogenous BDNF attenuates long-term sensorimotor behavior deficits after $S A H$. Next, the long-term sensorimotor behavior deficits at day 14 after SAH were evaluated using the adhesive removal task and Frey test. The time for taking on latency to start sticker removal (sensory function; Fig. 4A), the time to effective sticker removal (motor function; Fig. 4B) and the time for removing the adhesive from the left forepaw (impaired; Fig. 4C) were significantly increased in the $\mathrm{SAH}+$ vehicle group when compared with the sham group $(\mathrm{P}<0.05)$, while BDNF treatment significantly attenuated these increases (Fig. 4A-C). Furthermore, compared with the sham group, SAH caused impairment in mechanical sensitivity to 
A

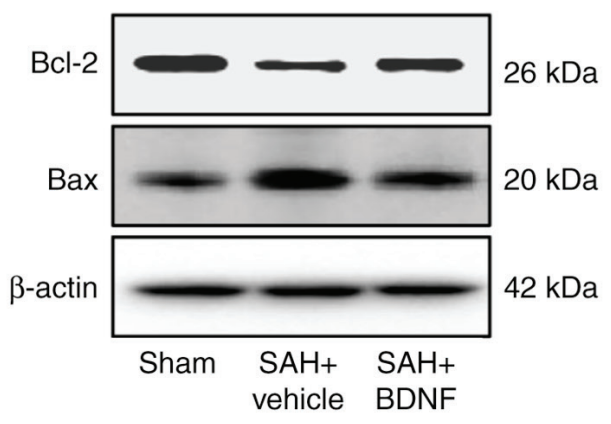

$\mathrm{B}$

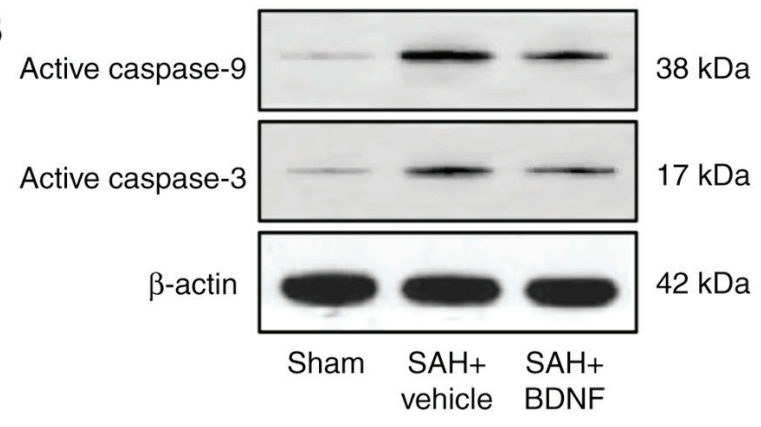

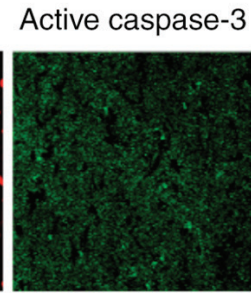
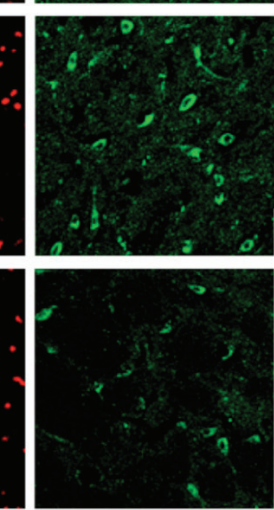

III
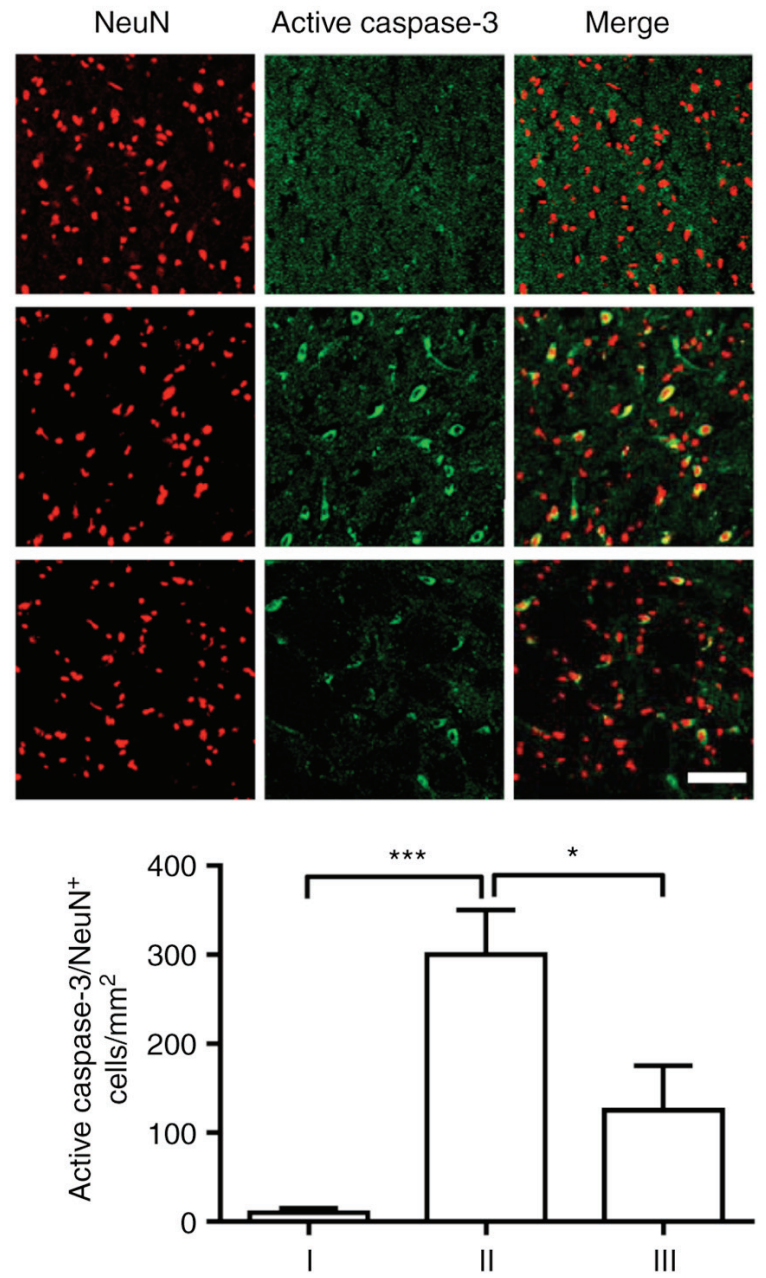
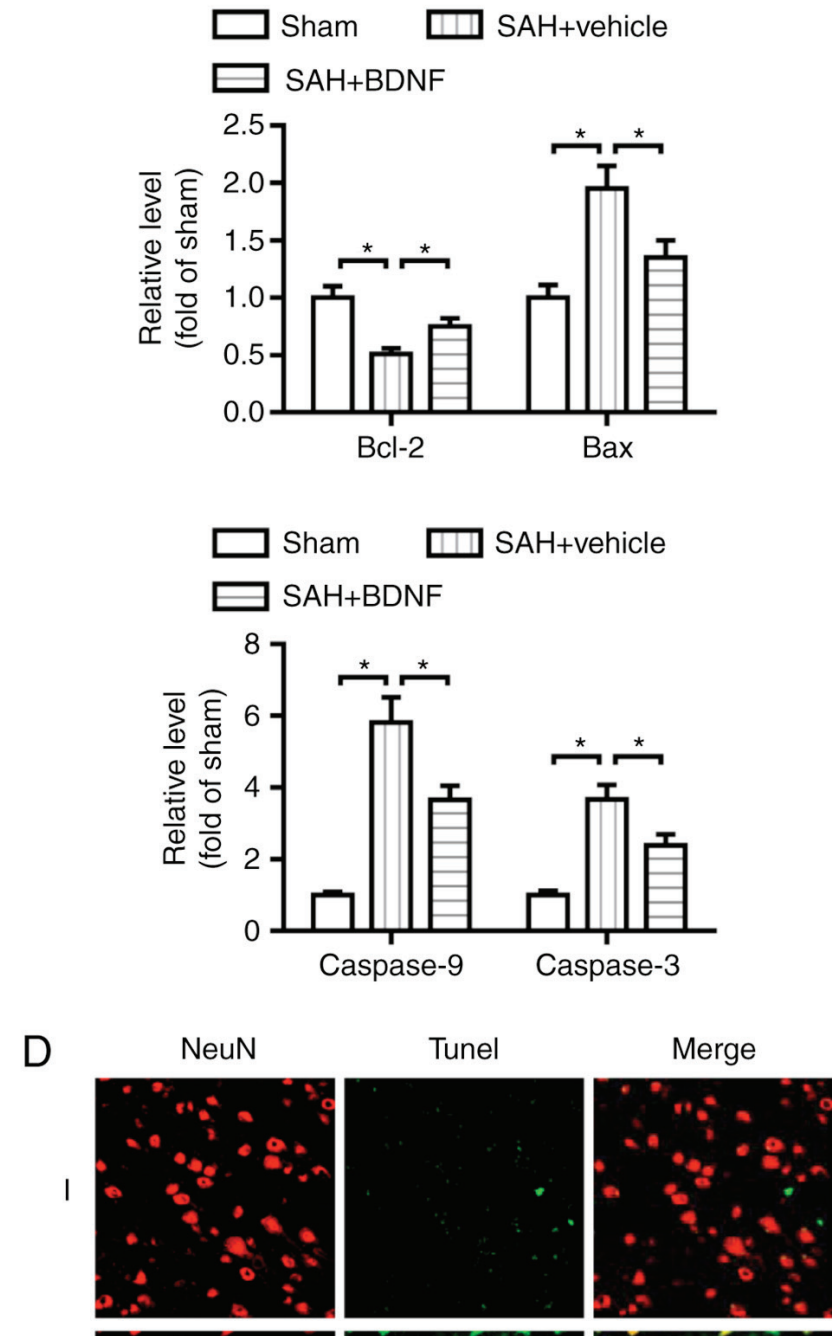

II
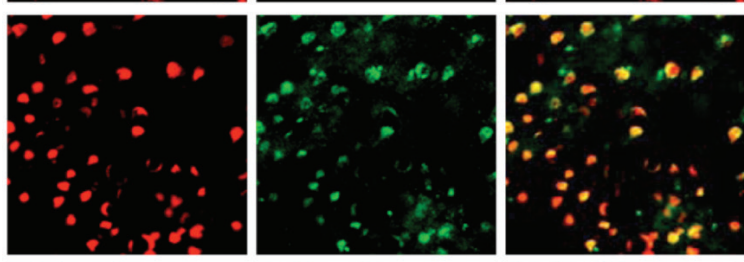

III
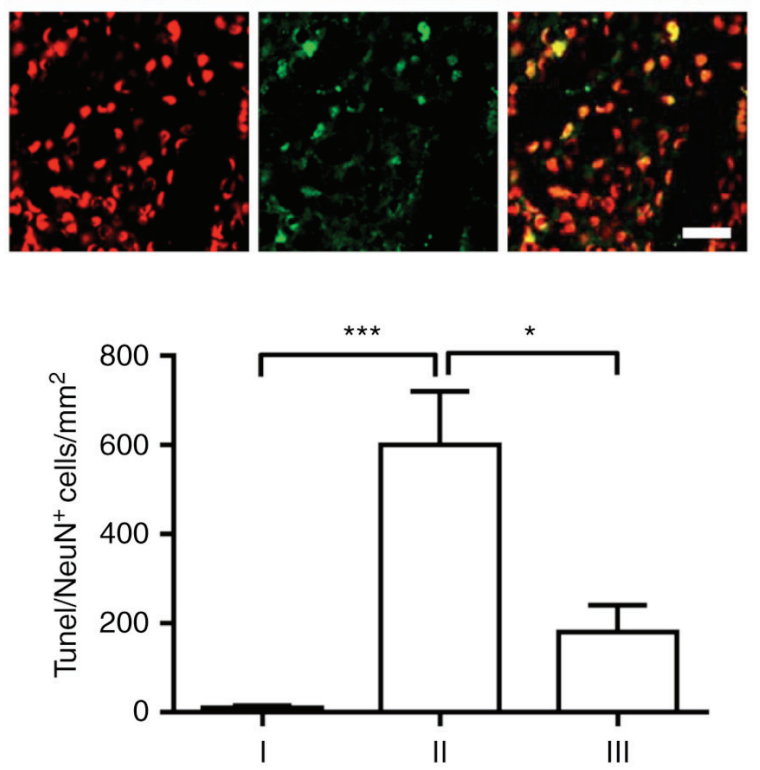

Figure 3. Exogenous BDNF attenuates neuronal apoptosis at $72 \mathrm{~h}$ after SAH. (A) Expression of Bcl-2 and Bax, (B) activation of caspase-9 and caspase-3, (C) activated caspase-3/NeuN staining-positive neurons and (D) Tunel/NeuN staining-positive neurons, in the basal cortex of the sham (I), SAH + vehicle (II) and SAH + BDNF (III) groups. (A-D) $\mathrm{P}=0.01,{ }^{*} \mathrm{P}<0.05$; (C-D) $\mathrm{P}=0.0009,{ }^{* * *} \mathrm{P}<0.001$; one-way analysis of variance comparison test, $\mathrm{n}=6$ in each group. Scale bar, $50 \mu \mathrm{m}$. BDNF, brain-derived neurotrophic factor; SAH, subarachnoid hemorrhage. 

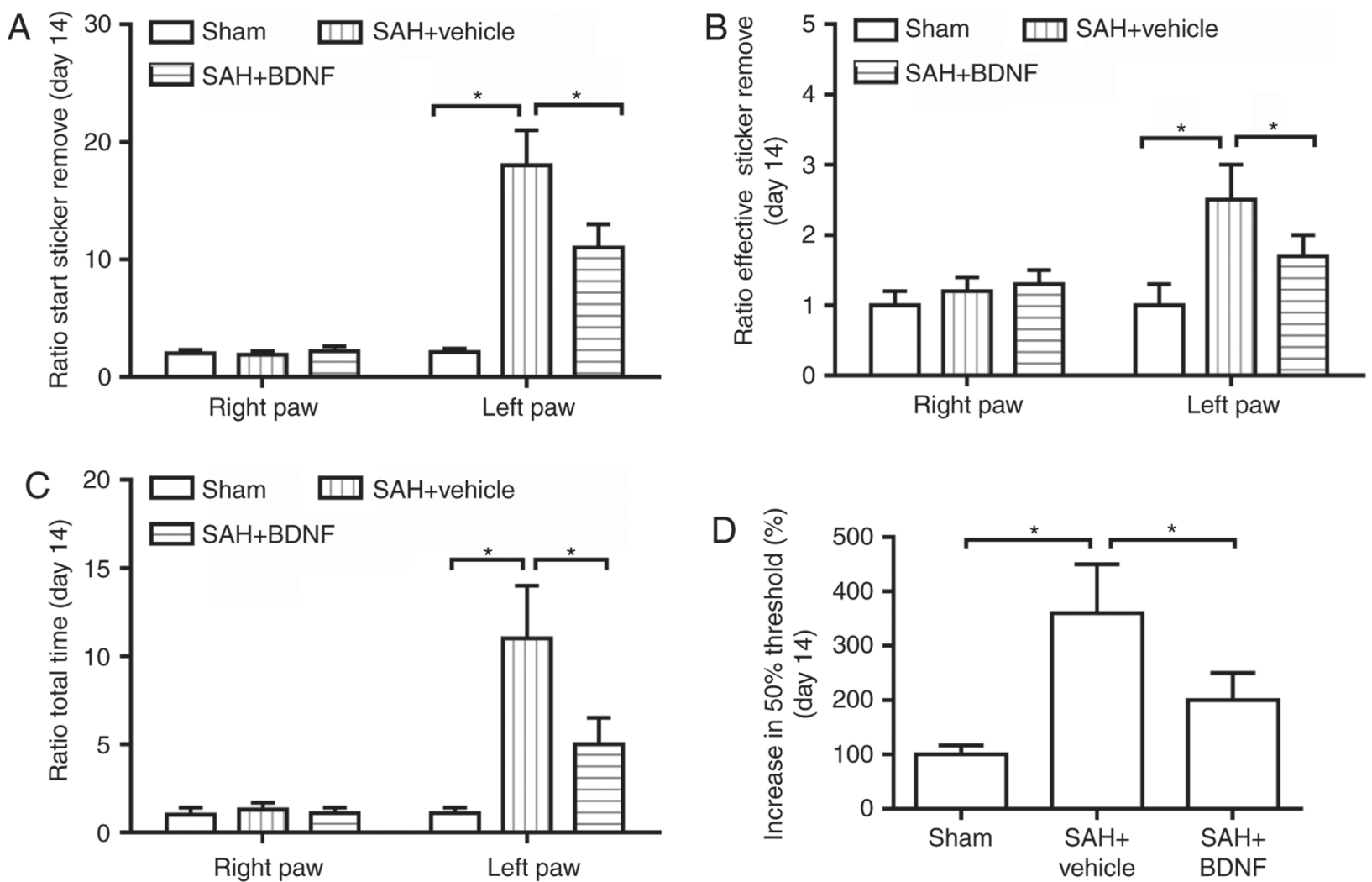

Figure 4. Exogenous BDNF attenuates long-term sensorimotor behavior deficits after SAH. (A-C) Sensorimotor function was assessed using the adhesive removal task at day 14 following SAH. The value was presented at 1 as compared with that observed in the sham group. (A) sensory input (latency to start sticker removal time), (B) motor behavior (effective sticker removal time), (C) total adhesive removal time, in the sham, $\mathrm{SAH}+\mathrm{vehicle}$ and $\mathrm{SAH}+\mathrm{BDNF}$ groups. (D) Mechanical sensitivity was evaluated using the von Frey test at day 14 following SAH. The $50 \%$ threshold was calculated in the sham, SAH + vehicle and $\mathrm{SAH}+\mathrm{BDNF}$ groups. $\mathrm{P}=0.01$ and ${ }^{*} \mathrm{P}<0.05$; one-way analysis of variance comparison test, $\mathrm{n}=6$ in each group. $\mathrm{BDNF}$, brain-derived neurotrophic factor; SAH, subarachnoid hemorrhage.

innocuous stimuli, while BDNF treatment significantly attenuated this impairment $(\mathrm{P}<0.05$; Fig. 4D), indicating that $\mathrm{BDNF}$ improved SAH-induced loss of sensory function.

\section{Discussion}

The present study demonstrated that ICV injection of BDNF attenuated neurobehavioral deficits and neuronal apoptosis, reduced the expression of Bax, activated caspase- 9 and caspase- 3 in the basal cortex in an endovascular perforation SAH model in rats. These findings suggest that exogenous BDNF is neuroprotective against brain injury after SAH, at least in part through an anti-apoptotic mechanism.

The expression level of BDNF following $\mathrm{SAH}$ has been poorly tested and the reported results are diverse. The concentration of BDNF in the cerebrospinal fluid was significantly decreased at day 3, while it increased markedly at days 5 and 7 after SAH (11). The BDNF level in the cerebral cortex significantly decreased at 4 and $48 \mathrm{~h}$ post-SAH $(10,12)$. There was no significant change in BDNF expression of the cerebral hemispheres at 4 and $24 \mathrm{~h}$ following SAH (9). In the present study, western blot analysis and ELISA revealed that the BDNF concentration of the basal cortex significantly decreased at 6, 12, 24, 48 and $72 \mathrm{~h}$ following SAH. In neurons, $\mathrm{N}$-methyl-D-aspartic acid triggers a $\mathrm{Ca}^{2+}$ signal and induces BDNF release $(24,25)$. As it has been reported that neuron apoptosis occurs very early in the basal cortex following
SAH (2), it was hypothesized that the downregulation of BDNF induced by SAH is possibly caused by neuronal apoptosis. Further studies are required to investigate the BDNF expression in short- and long-term brain damage, and elucidate whether inhibition of neuronal apoptosis enhances BDNF expression after $\mathrm{SAH}$.

In line with other studies using the modified Garcia score, beam balance test and rotarod test for evaluation of neurological score after SAH $(14,15,20,21)$, the present study observed neurological deficits following SAH, which improved by ICV injection of BDNF. Moreover, the adhesive removal task and Frey test were used to assess the long-term sensorimotor behavior deficit at day 14 after SAH (23). The results of the present study revealed that SAH rats exhibited impairment in tactile sensitivity and mechanical sensitivity. However, BDNF treatment restored these functions. Neuronal apoptosis is considered to play a key role in brain injury following $\mathrm{SAH}$ and is associated with neurological deterioration and poor outcome (2). The results of the present study demonstrated that ICV injection of BDNF reduced the SAH-related increased number of activated caspase-3- or TUNEL-positive neurons in the basal cortex. Although not tested in the present study, the anti-apoptotic mechanism of BDNF may be similar to that reported by previous studies $(8,9)$, where BDNF suppressed neuronal apoptosis through activation of the BDNF/TrkB signaling pathway after SAH. Accumulating evidence indicates that the balance between the anti-apoptotic protein Bcl-2 
and the pro-apoptotic protein Bax is closely associated with cell apoptosis and survival $(26,27)$. The increased Bax level leads to mitochondrial membrane permeabilization, which induces the mitochondrial release of pro-apoptotic factors, then promotes the initiator of caspase- 9 activation and consequently the effector of activated caspase-3 activation $(28,29)$. In the present study, decreased expression of Bcl-2 and increased expression of Bax after $\mathrm{SAH}$ was observed, but exogenous BDNF reversed these changes. The results of the present study also found that $\mathrm{SAH}$ induces activation of caspase- 9 and caspase-3, which is attenuated by ICV injection of BDNF. Therefore, exogenous BDNF appears to reduce neuronal apoptosis through regulation of $\mathrm{Bcl}-2$, Bax and caspase-3 expression.

In conclusion, the present study demonstrated that exogenous BDNF attenuated neuronal apoptosis through inhibiting caspase- 9 and caspase- 3 activation, and improved the neurological deficits and long-term behavioral deficits after SAH.

\section{Acknowledgements}

Not applicable.

\section{Funding}

The present study was supported by the National Science Foundation of China (grant no. 81170095).

\section{Authors' contributions}

HW designed experiments and wrote the manuscript. HC, YD, XL and JR performed the experiments and analyzed the results. All authors read and approved the final manuscript.

\section{Availability of data and materials}

The datasets used and/or analyzed during the current study are available from the corresponding author on reasonable request.

\section{Ethics approval and consent to participate}

Animal procedures were approved by the Animal Use and Care Committee of Hubei University of Medicine.

\section{Patient consent for publication}

Not applicable.

\section{Competing interests}

The authors declare that they have no competing interests.

\section{References}

1. Nieuwkamp DJ, Setz LE, Algra A, Linn FH, de Rooij NK and Rinkel GJ: Changes in case fatality of aneurysmal subarachnoid haemorrhage over time, according to age, sex, and region: A meta-analysis. Lancet Neurol 8: 635-642, 2009.

2. Hasegawa Y, Suzuki H, Sozen T, Altay O and Zhang JH: Apoptotic mechanisms for neuronal cells in early brain injury after subarachnoid hemorrhage. Acta Neurochir Suppl 110: 43-48, 2011
3. Baker-Herman TL, Fuller DD, Bavis RW, Zabka AG, Golder FJ, Doperalski NJ, Johnson RA, Watters JJ and Mitchell GS: BDNF is necessary and sufficient for spinal respiratory plasticity following intermittent hypoxia. Nat Neurosci 7: 48-55, 2004.

4. Bramham CR and Messaoudi E: BDNF function in adult synaptic plasticity: The synaptic consolidation hypothesis. Prog Neurobiol 76: 99-125, 2005.

5. Kowiański P, Lietzau G, Czuba E, Waśkow M, Steliga A and Moryś J: BDNF: A key factor with multipotent impact on brain signaling and synaptic plasticity. Cell Mol Neurobiol 38: 579-593, 2018.

6. Siironen J, Juvela S, Kanarek K, Vilkki J, Hernesniemi J and Lappalainen J: The Met allele of the BDNF Val66Met polymorphism predicts poor outcome among survivors of aneurysmal subarachnoid hemorrhage. Stroke 38: 2858-2860, 2007.

7. Vilkki J, Lappalainen J, Juvela S, Kanarek K, Hernesniemi JA and Siironen J: Relationship of the Met allele of the brain-derived neurotrophic factor Val66Met polymorphism to memory after aneurysmal subarachnoid hemorrhage. Neurosurgery 63: 198-203; discussion 203, 2008.

8. Tang J, Hu Q, Chen Y, Liu F, Zheng Y, Tang J, Zhang J and Zhang JH: Neuroprotective role of an N-acetyl serotonin derivative via activation of tropomyosin-related kinase receptor B after subarachnoid hemorrhage in a rat model. Neurobiol Dis 78: 126-133, 2015.

9. Hasegawa Y, Suzuki H, Altay O and Zhang JH: Preservation of tropomyosin-related kinase B (TrkB) signaling by sodium orthovanadate attenuates early brain injury after subarachnoid hemorrhage in rats. Stroke 42: 477-483, 2011.

10. Jiang Y, Liu DW, Han XY, Dong YN, Gao J, Du B, Meng L and Shi JG: Neuroprotective effects of anti-tumor necrosis factor-alpha antibody on apoptosis following subarachnoid hemorrhage in a rat model. J Clin Neurosci 19: 866-872, 2012.

11. Lee WD, Wang KC, Tsai YF, Chou PC, Tsai LK and Chien CL: Subarachnoid hemorrhage promotes proliferation, differentiation, and migration of neural stem cells via BDNF upregulation. PLoS One 11: e0165460, 2016.

12. Zhang ZY, Yang MF, Wang T, Li DW, Liu YL, Zhang JH and Sun BL: Cysteamine alleviates early brain injury via reducing oxidative stress and apoptosis in a rat experimental subarachnoid hemorrhage model. Cell Mol Neurobiol 35: 543-553, 2015.

13. Li M, Wang Y, Wang W, Zou C, Wang X and Chen Q: Recombinant human brain-derived neurotrophic factor prevents neuronal apoptosis in a novel in vitro model of subarachnoid hemorrhage. Neuropsychiatr Dis Treat 13: 1013-1021, 2017.

14. Sugawara T, Ayer R, Jadhav V and Zhang JH: A new grading system evaluating bleeding scale in filament perforation subarachnoid hemorrhage rat model. J Neurosci Methods 167: 327-334, 2008.

15. Zhang Z, Liu J, Fan C, Mao L, Xie R, Wang S, Yang M, Yuan H, Yang X, Sun J, et al: The GluN1/GluN2B NMDA receptor and metabotropic glutamate receptor 1 negative allosteric modulator has enhanced neuroprotection in a rat subarachnoid hemorrhage model. Exp Neurol 301: 13-25, 2018.

16. Zhang ZY, Sun BL, Liu JK, Yang MF, Li DW, Fang J, Zhang S, Yuan QL and Huang SL: Activation of mGluR5 attenuates microglial activation and neuronal apoptosis in early brain injury after experimental subarachnoid hemorrhage in rats. Neurochem Res 40: 1121-1132, 2015.

17. Zhu Q, Enkhjargal B, Huang L, Zhang T, Sun C, Xie Z, Wu P, Mo J, Tang J, Xie Z and Zhang JH: Aggf1 attenuates neuroinflammation and BBB disruption via PI3K/Akt/NF- $\mathrm{BB}$ pathway after subarachnoid hemorrhage in rats. J Neuroinflammation 15: $178,2018$.

18. Guan J, Zhang B, Zhang J, Ding W, Xiao Z, Zhu Z, Han Q, Wu C, Sun $Y$, Tong W, et al: Nerve regeneration and functional recovery by collagen-binding brain-derived neurotrophic factor in an intracerebral hemorrhage model. Tissue Eng Part A 21: 62-74, 2015.

19. Li H, Yu JS, Zhang DD, Yang YQ, Huang LT, Yu Z, Chen RD, Yang HK and Hang CH: Inhibition of the receptor for advanced glycation end-products (RAGE) attenuates neuroinflammation while sensitizing cortical neurons towards death in experimental subarachnoid hemorrhage. Mol Neurobiol 54: 755-767, 2017.

20. Huang CY, Wang LC, Wang HK, Pan CH, Cheng YY, Shan YS, Chio CC and Tsai KJ: Memantine alleviates brain injury and neurobehavioral deficits after experimental subarachnoid hemorrhage. Mol Neurobiol 51: 1038-1052, 2015. 
21. Zhou YD and Cai L: Calpeptin reduces neurobehavioral deficits and neuronal apoptosis following subarachnoid hemorrhage in rats. J Stroke Cerebrovasc Dis 28: 125-132, 2019.

22. Friedrich V, Flores R and Sehba FA: Cell death starts early after subarachnoid hemorrhage. Neurosci Lett 512: 6-11, 2012.

23. Kooijman E, Nijboer $\mathrm{CH}$, van Velthoven CT, Mol W, Dijkhuizen RM, Kesecioglu J and Heijnen CJ: Long-term functional consequences and ongoing cerebral inflammation after subarachnoid hemorrhage in the rat. PLoS One 9: e90584, 2014.

24. Jiang X, Zhu D, Okagaki P, Lipsky R, Wu X, Banaudha K, Mearow K, Strauss KI and Marini AM: N-methyl-D-aspartate and TrkB receptor activation in cerebellar granule cells: An in vitro model of preconditioning to stimulate intrinsic survival pathways in neurons. Ann N Y Acad Sci 993: 134-145; discussion 159-160, 2003.

25. Zhu D, Wu X, Strauss KI, Lipsky RH, Qureshi Z, Terhakopian A, Novelli A, Banaudha K and Marini AM: N-methyl-D-aspartate and TrkB receptors protect neurons against glutamate excitotoxicity through an extracellular signal-regulated kinase pathway. J Neurosci Res 80: 104-113, 2005.
26. Wang X, He X, Hu S, Sun A and Lu C: Involvement of Bim in Photofrin-mediated photodynamically induced apoptosis. Cell Physiol Biochem 35: 1527-1536, 2015.

27. Willis S, Day CL, Hinds MG and Huang DC: The Bcl-2-regulated apoptotic pathway. J Cell Sci 116: 4053-4056, 2003.

28. Hong Y, Shao A, Wang J, Chen S, Wu H, McBride DW, Wu Q, Sun $\mathrm{X}$ and Zhang J: Neuroprotective effect of hydrogen-rich saline against neurologic damage and apoptosis in early brain injury following subarachnoid hemorrhage: Possible role of the Akt/GSK3 $\beta$ signaling pathway. PLoS One 9: e96212, 2014.

29. Xie Z, Enkhjargal B, Wu L, Zhou K, Sun C, Hu X, Gospodarev V, Tang J, You C and Zhang JH: Exendin-4 attenuates neuronal death via GLP-1R/PI3K/Akt pathway in early brain injury after subarachnoid hemorrhage in rats. Neuropharmacology 128: 142-151, 2018.

This work is licensed under a Creative Commons Attribution-NonCommercial-NoDerivatives 4.0 International (CC BY-NC-ND 4.0) License. 